RCM

Letter to the Editor

To the Editor-in-Chief

Sir,

\section{Hyperhydroxyprolinaemia: a new case diagnosed during neonatal screening with tandem mass spectrometry}

Hydroxyproline oxidase deficiency (MIM 237000) is a rare autosomal recessive defect in the catabolism of the imino acid hydroxyproline derived from endogenous collagen turnover and the breakdown of dietary collagen. The most important biochemical finding is an increase in the hydroxyproline plasmatic level with an abnormal urinary excretion. Scanty data report ambiguous information about clinical involvement, such as mental retardation or visual perceptual dysfunction; ${ }^{1,2}$ they seem, therefore, to be possibly due to analytical bias. ${ }^{3} \mathrm{Up}$ to now only one case of hydroxyprolinaemia diagnosed during newborn screening has been reported. ${ }^{4}$

During expanded newborn screening in Tuscany using tandem mass spectrometry (MS/MS), 5,6 we diagnosed a newborn with hydroxyprolinaemia. The patient, a male, was born at term (A.I. $9^{\mathrm{I}}-10^{\mathrm{V}}$ ) to non-consanguineous parents from Tuscany, with a weight of $2980 \mathrm{~g}$ after a pregnancy during which diabetes was treated with insulin. No clinical abnormalities were found in his first days of life. His dried blood spot was collected on the third day of life and analysed during the fourth day.

The dried blood spot was punched into a micro 96-well plate, and $200 \mu \mathrm{L}$ of methanol containing labeled acylcarnitines and amino acid standards were added. The sample was shaken on a vortex system for $20 \mathrm{~min}$. After shaking, the extracted solution of amino acids and acylcarnitines was transferred to a new micro 96-well plate, and was then dried under a nitrogen flow at $50^{\circ} \mathrm{C}$. The extracted acylcarnitines and amino acids were derivatised to butyl esters using n-butanol with $\mathrm{HCl}(3 \mathrm{M})$ at $65^{\circ} \mathrm{C}$ for $25 \mathrm{~min}$. After derivatisation the sample was dried under a nitrogen flow at $55^{\circ} \mathrm{C}$ and then redissolved in $200 \mu \mathrm{L}$ of an aqueous solution of $50 \%$ acetonitrile containing $0.1 \%$ formic acid (mobile phase) and $40 \mu \mathrm{L}$ of the diluted sample were injected using flow injection analysis (FIA) for the MS/MS experiments.

An Applied Biosystems-MDS Sciex (Toronto, Canada) API 4000 triplequadrupole mass spectrometer equipped with a TurbolonSpray source was employed for this study. The TurbolonSpray source was operated in positive ion mode with a needle potential of $+5900 \mathrm{~V}$ and with a "Turbo" gas flow of $10 \mathrm{~L} / \mathrm{min}$ of air heated at $150^{\circ} \mathrm{C}$ (nominal heating-gun temperature). Mass calibration and resolution adjustments on the resolving quadrupoles were performed automatically using a PPG solution $\left(10^{-4} \mathrm{~mol} / \mathrm{L}\right)$ introduced via the builtin infusion pump. The peak width was set on both resolving quadrupoles at $0.7 \mathrm{Th}$ (measured at $1 / 2$ height) for all MS and MS/MS experiments. Collisionally activated dissociation (CAD) MS/MS was performed in the LINAC Q2 collision cell, operating with 10 mTorr pressure of nitrogen as collision gas. The declustering potential (DP) and collision energy (CE) were automatically optimised for amino acids using the Analyst 1.4 software. The resulting DP was $+20 \mathrm{~V}$, and optimal CE was found to be $32 \mathrm{eV}$ (laboratory frame) in the case of amino acids.

MS and MS/MS spectra were collected in continuous flow mode by connecting the infusion pump directly to the TurboIonSpray source. For these optimisation experiments, a standard solution of $10 \mathrm{ng} / \mu \mathrm{L}$ of each amino acid in an aqueous solution of $50 \%$ acetonitrile containing $0.1 \%$ formic acid was infused at $10 \mu \mathrm{L} / \mathrm{min}$.

The quantitation experiments were performed using a series 1100 Agilent Technologies (Waldbronn, Germany) CapPump coupled to an Agilent Micro $\mu \mathrm{WS}$ autosampler, both fully controlled from the API 4000 data system.
The flow rate was $50 \mu \mathrm{L} / \mathrm{min}$ using an aqueous solution of $50 \%$ acetonitrile containing $0.1 \%$ formic acid. The eluent from the high-performance liquid chromatography (HPLC) system was directed to the TurboIonSpray probe. The acquired data were processed using the Analyst 1.4 proprietary software including the "Explore" option (for chromatographic and spectral interpretation) and the ChemoView software (for quantitative information generation).

The amino acid analysis was performed using a neutral loss scan in the $m / z$ range $130-280$. In this case, the leucine/isoleucine/hydroxyproline MS/MS signal $(\mathrm{m} / \mathrm{z} 188.2 \rightarrow 86.1$, i.e. a neutral loss of $102.1 \mathrm{Da})$ was unusually high (Fig. 1), indicating a concentration of $390 \mu \mathrm{mol} / \mathrm{L}$ (normal values (n.v.) 60-195, calculated as the average $\pm 2 \times$ standard deviation (SD) from $\sim 5000$ newborn dried blood spots) for the newborn spot, and to $412 \mu \mathrm{mol} / \mathrm{L}$ for the analysis of a subsequent sample taken at 6 days of age. The labelled internal standard was ${ }^{2} \mathrm{H}_{3}$ leucine $(m / z \quad 191.2 \rightarrow 89.1)$. The dried urine spot analysed by the above MS/MS screening method ${ }^{5,6}$ for dried blood spots confirmed abnormal levels up to $2742 \mu \mathrm{mol} / \mathrm{mmol}$ creatinine (n.v. $0-143)$. Maple syrup urine disease (MSUD, MIM 248600), a metabolic disorder affecting the metabolism of branched-chain amino acids, ${ }^{7}$ was immediately excluded because no abnormal valine level was detected.

Only two cases of hyperleucinisoleucinemia that were not associated with the more common MSUD (normal valine levels), but were associated with hyperprolinemia type II, have been described previously; ${ }^{8}$ these two defects appear to be not correlated. However, in our case, quantitative amino acid detection, using an HPLC-based amino acid analyser (Biochrom20 plus, Manchester, UK), confirmed an abnormal hydroxyproline plasma level of $339 \mu \mathrm{mol} / \mathrm{L}$ (n.v. 16-53) and urinary excretion of $2960 \mu \mathrm{mol} / \mathrm{mmol}$ creatinine (n.v. 0-143). The patient was clinically normal at 10th day of age, and at 4 months the psychomotor developmental, visual evocated potentials, and fundus oculi were normal. It was not possible to confirm the 

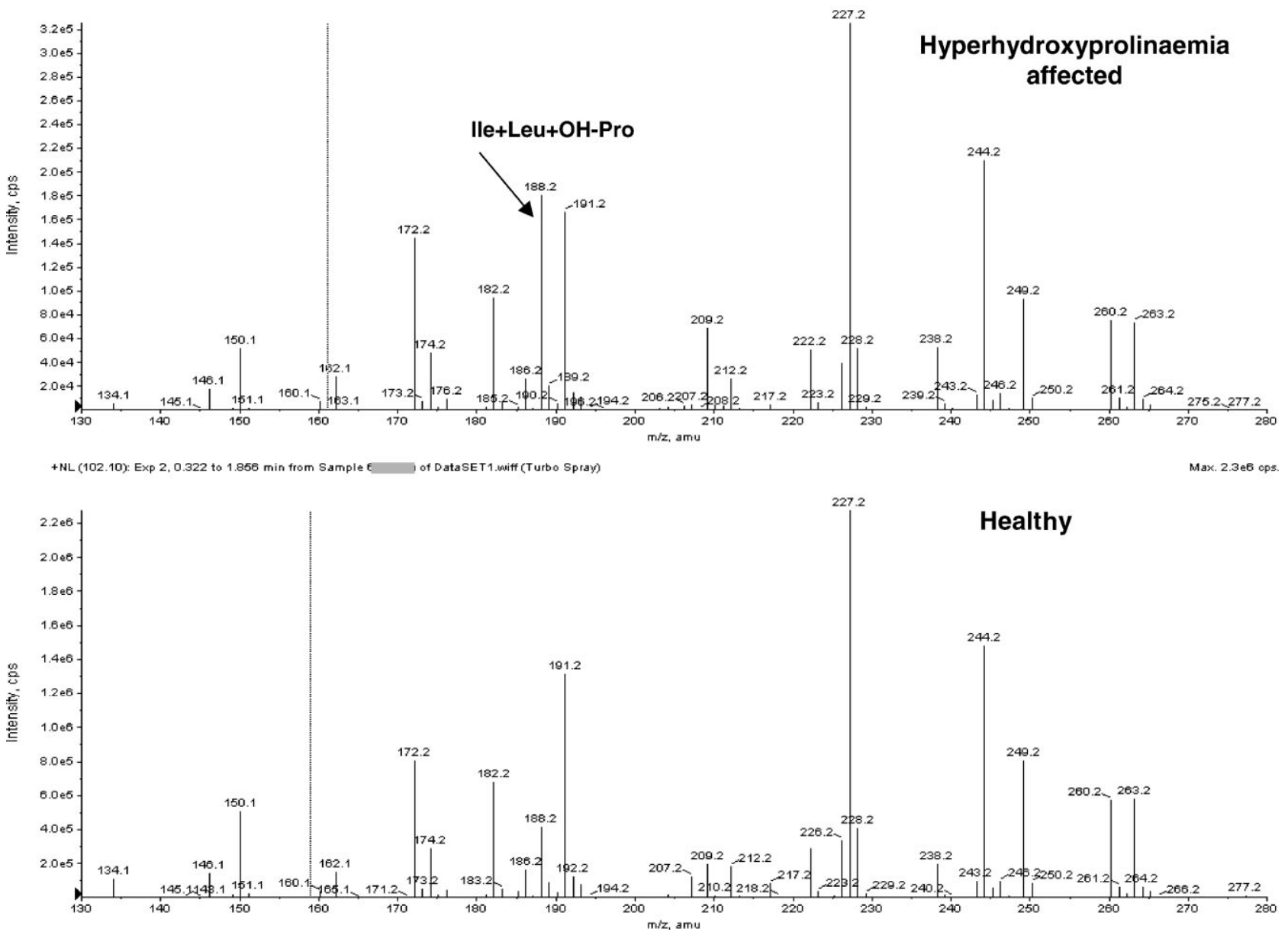

Figure 1. Normal amino acid profile compared with one from a patient with hyperhydroxyprolinaemia.

diagnosis by genomic analysis since, to our knowledge, the hydroxyproline oxidase gene has not yet been characterized.

Profiles of acylcarnitines and amino acids, obtained during newborn screening with LC/MS/MS, permits detection of more than 30 inborn errors of metabolism. However, in some cases, absence of specificity can be a limitation. For example, drug interferences on acylcarnitine profiles have been reported by Abdenur et al. ${ }^{9}$ In the present case, butyl esters of leucine, isoleucine and hydroxyproline are isobaric, with the same $m / z$ value (188.2) for the $[\mathrm{M}+\mathrm{H}]^{+}$ions and for their major fragment ions (86.1), making their differentiation by MS/MS impossible.

Therefore, it possible to draw a general conclusion that this report confirms that, in some cases, newborn screening with tandem mass spectrometry is only the first diagnostic approach, and that further confirmatory analytical tests are indispensable to confirm the original diagnosis.

Giancarlo la Marca*, Sabrina Malvagia, Elisabetta Pasquini, Maria Alice Donati, Serena Gasperini, Elena Procopio and Enrico Zammarchi

Metabolic and Muscular Unit, Department of Pediatrics, University of Florence, Meyer Children's Hospital, Florence, Italy

*Correspondence to: G. la Marca, Department of Pediatrics, Meyer Children's Hospital, Via Luca Giordano 13, 50132 Florence, Italy.

E-mail:g.lamarca@meyer.it

\section{REFERENCES}

1. Efron ML, Bixby EM, Pryles CV. New Eng. J. Med. 1965; 272: 1299.

2. Kim SZ, Varvogli L, Waisbren SE, Levy HL. J. Pediatr. 1997; 130: 437
3. Phang JM, Scriver CR. In The Metabolic Basis of Inherited Disease (8th edn), vol. I, Scriver CR, Beaudet AL, Sly WS, Valle D (eds). McGraw-Hill: New York, 2001; 1833-1834.

4. Baykal T, Karaaslan I, Gokcay G, Demir F, Laleli Y, Demirkol M. J. Inherit. Metab. Dis. 2004; 27: 781.

5. Millington DS, Kodo N, Norwood DL, Roe CR. J. Inherit. Metab. Dis. 1990; 13: 321.

6. Millington DS, Terada N, Kodo N, Chace DH. Advancesin Chemical Diagnosis and Treatment of Metabolic Disorders, vol. 1. John Wiley: New York, 1992; 59-71.

7. Westall RG, Dancis J, Miller S. Am. J. Dis. Child 1957; 94: 571

8. Jeune M, Collombel C, Michel M, et al. Ann. Pediatr. 1970; 17: 349.

9. Abdenur JE, Chamoles NA, Guinle $\mathrm{AE}$, Schenone $\mathrm{AB}$, Fuertes AN. J. Inherit. Metab. Dis. 1998; 21: 624.

Received 5 January 2005 Revised 24 January 2005 Accepted 25 January 2005 